\title{
STABILITY PROPERTIES OF FLOWS WITH PURE POINT SPECTRUM
}

\author{
BRUNO SCARPELLINI
}

\section{Introduction}

In this note we show that a large class of measure preserving flows with pure point spectrum have some Ljapounov-like stability properties. We discuss the problem by looking at an example. Let $\Omega=\left\{(x, y): x^{2}+y^{2} \leqslant 1\right\}$ be the unit disc, let $(r, \phi)$ be the polar coordinates of $(x, y) \in \Omega$ and let $\mu$ be Lebesgue measure, restricted to $\Omega$ and normalized to $\mu(\Omega)=1$. On $\Omega$ we define two flows $S_{t}, T_{t}, t \in \mathbb{R}$ (where $\mathbb{R}$ denotes the reals) by the conditions $S_{t}((r, \phi))=(r, \phi+t)$ and $T_{t}((r, \phi))=(r, \phi+r t)$ respectively. Then we have two measure preserving dynamical systems: $D_{1}=\left(\Omega, S_{t}, t \in \mathbb{R}, \mu\right)$, and $D_{2}=\left(\Omega, T_{t}, t \in \mathbb{R}, \mu\right)$ which have the common property

(A) if $f \in C(\Omega)$ then $f\left(P_{t}\right), t \in \mathbb{R}$ is periodic (where $P_{t}=S_{t}(P)$ or $P_{t}=T_{t}(P)$ according to the system under consideration).

However $D_{1}$ has pure point spectrum while $D_{2}$ has not and $D_{1}$ is Ljapounov stable while $D_{2}$ is not. In order to look at a similar but more general situation we replace condition (A) by

(B) if $f \in C(\Omega)$ then for almost all points $P$ the function $f\left(P_{t}\right), t \in \mathbb{R}$ is Besicovitch almost periodic (this is explained in §2).

Next let $d(P, Q)$ be the Euclidean distance of $P, Q$ and put

$$
d^{\prime}(P, Q)=\lim \sup _{T}\left((2 T)^{-1} \int_{-T}^{+T} d\left(P_{t}, Q_{t}\right)^{2} d t\right)^{1 / 2} .
$$

A set $M \subseteq \Omega$ is called $B$-stable if for $\varepsilon>0$ there is $\delta>0$ such that $P, Q \in M$ and $d(P, Q) \leqslant \delta$ imply that $d^{\prime}(P, Q) \leqslant \varepsilon$. Now consider

(C) for every $\varepsilon>0$ there is a closed set $M \subseteq \Omega$ with $\mu(M) \geqslant 1-\varepsilon$ which is $B$-stable.

It is clear that every Ljapounov stable system (and thus in particular $D_{1}$ ) satisfies condition (C); we simply take $M=\Omega$ for all $\varepsilon>0$. It turns out that for a large class of measure preserving flows properties (B) and (C) are necessary and sufficient for the flow to have pure point spectrum. This fact will follow from similar but more general statements whose exact formulations are given later. For further comments we refer to the final paragraph. 


\section{Preliminaries}

(a) Henceforth, $\Omega$ is a compact metric space with metric $d$, and $\mu$ is a probability measure on the Borel field induced by the topology of $\Omega$ which is then automatically regular $\left[4\right.$, p. 223]. A group $S_{t}, t \in \mathbb{R}$ of measure preserving automorphisms of $\Omega$ is given such that the mapping $(P, t) \rightarrow S_{t}(P)$ from $\Omega \times \mathbb{R}$ to $\Omega$ is measurable. In addition we assume the group $S_{t}, t \in \mathbb{R}$ to have the following property:

(I) if $M \subseteq \Omega$ is a closed set then for every closed interval $[a, b]$ the set $\bigcup M_{t}, t \in[a, b]$ is a Borel set.

The triple $F=\left(\Omega, S_{t}, t \in \mathbb{R}, \mu\right)$ is referred to as a flow.

(b) Use will be made of the ergodic decomposition theory of BogoljubovKrylov $[2,11]$. Thus $U_{T} \subseteq \Omega$ is the set of transitive points which has the following well known properties (see, for example [11] for details):

(1) $U_{T}$ is invariant against $S_{t}, t \in \mathbb{R}$ and $\mu\left(U_{T}\right)=1$,

(2) with $P \in U_{T}$ is associated a Borel measure $\mu_{P}$ on $\Omega$ given by

$$
\int f d \mu_{P}=\lim (2 T)^{-1} \int_{-T}^{+T} f\left(P_{t}\right) d t
$$

for all $f \in C(\Omega)$,

(3) $\mu_{P}$ is invariant and ergodic with respect to $S_{t}, t \in \mathbb{R}$ and $\mu_{P}=\mu_{Q}$ if $Q=S_{t}(P)$ for some $t \in \mathbb{R}$,

(4) if $f \in L_{2}(\Omega, \mu)$ then for almost all $P \in U_{T}$ we have

$$
\begin{gathered}
f \in L_{2}\left(\Omega, \mu_{P}\right) \\
\int f d \mu=\int d \mu \int f d \mu_{P} \\
\int f d \mu_{P}=\lim (2 T)^{-1} \int_{-T}^{+T} f\left(P_{t}\right) d t
\end{gathered}
$$

For additional remarks on the theory of Bogoljubov-Krylov we refer to the final paragraph.

(c) The flow $F=\left(\Omega, S_{t}, t \in \mathbb{R}, \mu\right)$ induces a continuous unitary group $U_{t}, t \in \mathbb{R}$ on $L_{2}(\Omega, \mu)$. Likewise there is associated with each $P \in U_{T}$ a flow $F_{p}=\left(\Omega, S_{t}, t \in \mathbb{R}, \mu_{P}\right)$ which induces a unitary group on $L_{2}\left(\Omega, \mu_{P}\right)$, which, for notational simplicity is also denoted by $U_{t}, t \in \mathbb{R}$.

(d) For later use we collect some known facts about flows with point spectrum. 
First, let $U_{t}, t \in \mathbb{R}$ be a continuous unitary group on a Hilbert space $\mathbf{H}$. We call $f \in \mathbf{H}$ compact with respect to this group if for every sequence $t_{n} \in \mathbb{R}, n=1,2, \ldots$ there is a subsequence $t_{j(n)}, n=1,2, \ldots$ such that $U_{t_{j(n)}} f, n=1,2, \ldots$ is a Cauchy sequence. For $f \in \mathbf{H}$ let $\mathbf{H}(f) \subseteq \mathbf{H}$ be the closed, invariant subspace spanned by the elements $U_{t} f, t \in \mathbb{R}$. The following then holds:

(II) $U_{t}, t \in \mathbb{R}$, restricted to $\mathbf{H}(f)$, has pure point spectrum if and only if $f$ is compact.

Next, call an expression $T(t)=\sum_{1}^{n} \alpha_{j} \exp \left(i \lambda_{j} t\right),\left(\lambda_{j} \in \mathbb{R}, j=1, \ldots, n\right)$ a trigonometric polynomial. A function $f(t), t \in \mathbb{R}$ is called Besicovitch almost periodic if (i) $f$ is locally $L_{2}$ and (ii) there is a sequence $T_{n}(t)$ of trigonometric polynomials such that $\lim (2 T)^{-1} \int_{-T}^{+T}\left|f(t)-T_{n}(t)\right|^{2} d t$ exists and tends to 0 as $n \rightarrow \infty$. For a detailed discussion we refer to [16]. For $P \in U_{T}$ and $f \in L_{2}\left(\Omega, \mu_{P}\right)$ let $\mathbf{H}_{P}(f) \subseteq L_{2}\left(\Omega, \mu_{P}\right)$ be the closed invariant subspace spanned by the elements $U_{t} f, t \in \mathbb{R}$. The following is known:

(III) for almost all $P \in U_{T}$ the restriction of $U_{t}, t \in \mathbb{R}$ (acting on $L_{2}\left(\Omega, \mu_{P}\right)$ ) to $\mathbf{H}_{P}(f)$ has pure point spectrum if and only if the trajectory $f\left(P_{t}\right), t \in \mathbb{R}$ is Besicovitch almost periodic.

Proofs are given in $[16 ; 14$, Chapter $1, \S 2]$. There is a further result, also proved in [16], which connects (II) and (III), namely:

(IV) if $U_{t}, t \in \mathbb{R}$, acting on $L_{2}(\Omega, \mu)$, has pure point spectrum in $\mathbf{H}(f)=\operatorname{Span}\left\{U_{t} f, t \in \mathbb{R}\right\}$ then for almost all $P \in U_{T}$ the trajectory $f\left(P_{t}\right), t \in \mathbb{R}$ is Besicovitch almost periodic.

(d) We conclude with some notation. We write $P_{t}$ in place of $S_{t}(P)$. For $f \in L_{2}(\Omega, \mu)$ we define $f_{t}$ by $f_{t}(P)=f\left(P_{t}\right)$. The set of bounded Borel functions on $\Omega$ is denoted by $\mathrm{Bb}(\Omega)$. Finally, $\chi_{M}$ is the characteristic function of $M \subseteq \Omega$.

\section{Sufficient conditions for pure point spectrum}

In this section we give sufficient conditions for a flow to have pure point spectrum. Henceforth $F=\left(\Omega, S_{t}, t \in \mathbb{R}, \mu\right)$ is a fixed flow. We start with two definitions. In order to formulate the first we put

$$
D(f / P, Q)=\lim \sup _{T}\left\{(2 T)^{-1} \int_{-T}^{+T}\left|f\left(P_{t}\right)-f\left(Q_{t}\right)\right|^{2} d t\right\}^{1 / 2}
$$

Definition 1. Let $f \in L_{2}(\Omega, \mu)$ be bounded. A Borel set $M$ is called B-stable with respect to $f$ if for every $\varepsilon>0$ there is a $\delta>0$ with the property that

$$
\text { if } P, Q \in M \text { and } d(P, Q) \leqslant \delta \text { then } D(f / P, Q) \leqslant \varepsilon .
$$


Remark. The triangle inequalities $D\left(f / P_{1}, P_{3}\right) \leqslant D\left(f / P_{1}, P_{2}\right)+D\left(f / P_{2}, P_{3}\right)$ and $D(f+g / P, Q) \leqslant D(f / P, Q)+D(g / P, Q)$ are easily verified.

Definition 2. A point $P \in U_{T}$ is called compact with respect to $f \in \mathrm{Bb}(\Omega)$ if $f$ is a compact element with respect to the unitary group $U_{t}, t \in \mathbb{R}$ on $L_{2}\left(\Omega, \mu_{P}\right)$.

Next, let $M \subseteq U_{T}$ be a closed set with $\mu(M)>0$ and put $M_{t}=\left\{P_{t}: P \in M\right\}$. The set $M^{*}=\bigcup M_{t}, t \in \mathbb{R}$ is an invariant Borel set and its characteristic function $\chi_{M^{*}}$ is invariant: $\chi_{M^{*}}\left(P_{t}\right)=\chi_{M^{*}}(P)$. With $f \in C(\Omega)$ we associate the closed invariant subspace $\mathbf{H}\left(\chi_{M^{*}} f\right) \subseteq L_{2}(\Omega, \mu)$, spanned by the elements $f_{t} \chi_{M^{*}}, t \in \mathbb{R}$. Since $\mathbf{H}\left(\chi_{M^{*}} f\right)$ is invariant under $U_{t}, t \in \mathbb{R}$, we can look for the spectrum of $U_{t}, t \in \mathbb{R}$, restricted to the space $\mathbf{H}\left(\chi_{M^{*}} f\right)$. In particular we can ask for sufficient conditions to imply that this spectrum is a pure point spectrum. Such conditions are contained in the following theorem.

THEOREM 1. Assume that $f \in C(\Omega)$. Let $M \subseteq U_{T}$ be a closed set with $\mu(M)>0$. Assume that $M$ is $B$-stable with respect to $f$ and that every $P \in M$ is compact with respect to $f$. Then $U_{1}, t \in \mathbb{R}$, restricted to the subspace $\mathbf{H}\left(\chi_{M^{*}} f\right)$, has pure point spectrum.

Proof. By (1I) in $\$ 2$ the theorem is proved if we can show that $\chi_{M^{*}} f$ is compact. In order to prove this we proceed in steps.

(S1) We consider an arbitrary sequence $t_{n} \in \mathbb{R}, n=1,2, \ldots$. Our aim is to find a subsequence $s_{n} \in \mathbb{R}, n=1,2, \ldots$ of $t_{n}, n=1,2, \ldots$ such that $f_{s_{n}} \chi_{M^{*}}$ is a Cauchy sequence in $L_{2}(\Omega, \mu)$. To this end we take an arbitrary but fixed denumerable dense subset $P_{1}, P_{2}, \ldots \in M$. Since each $P_{j}$ is compact with respect to $f$ we find a double sequence $t_{n j}, n, j=1,2, \ldots$ such that (a) $t_{11}, t_{12}, \ldots$ is a subsequence of $t_{1}, t_{2}, \ldots$, (b) $t_{n+1,1}, t_{n+1,2}, \ldots$ is a subsequence of $t_{n 1}, t_{n 2}, \ldots$ and (c) $f_{t_{n 1}}, f_{t_{n 2}}, \ldots$ is a Cauchy sequence with respect to $L_{2}\left(\Omega, \mu_{P_{n}}\right)$ for $n=1,2, \ldots$. We now put $s_{n}=t_{n n}, n=1,2, \ldots$ and infer by the usual diagonal procedure that (d) $s_{1}, s_{2}, \ldots$ is a subsequence of $t_{1}, t_{2}, \ldots$ and $f_{s_{1}}, f_{s_{2}}, \ldots$ is a Cauchy sequence with respect to each Hilbert space $L_{2}\left(\Omega, \mu_{P_{n}}\right), n=1,2, \ldots$.

(S2) The next step consists in the verification that $f_{s_{1}}, f_{s_{2}}, \ldots$ is a Cauchy sequence with respect to $L_{2}\left(\Omega, \mu_{Q}\right)$ for every $Q \in M$. To this end we note that $D(f / P, Q)$ is translation invariant: $D(f / P, Q)=D\left(f / P_{t}, Q_{t}\right)$. Thus we fix a $Q \in M$ and an $\varepsilon>0$. Since $M$ is $B$-stable with respect to $f$ there is a $\delta>0$ such that $P \in M$, and $d(P, Q) \leqslant \delta$ implies that $D(f / P, Q) \leqslant \varepsilon / 4$. Since $P_{n}, n=1,2, \ldots$ is dense in $M$ we can find a $P=P_{n}$ such that $d(P, Q) \leqslant \delta$. Since $P$ is compact with respect to $f$ we can find an $N$ such that $j, k \geqslant N$ implies that $\left\|f_{s_{j}}-f_{s_{h}}\right\|_{P} \leqslant \varepsilon / 4$, where \|\|$_{P}$ is the Hilbert space norm in $L_{2}\left(\Omega, \mu_{P}\right)$. For $j, k \geqslant N$ we now have

$$
\begin{aligned}
\left\|f_{s_{j}}-f_{s_{k}}\right\|_{Q} & =\left\{\int\left|f_{s_{j}}-f_{s_{k}}\right|^{2} d \mu_{Q}\right\}^{1 / 2} \\
& =\lim _{T}\left\{(2 T)^{-1} \int_{-T}^{+T}\left|f\left(Q_{s_{j}+t}\right)-f\left(Q_{s_{k}+t}\right)\right|^{2} d t\right\}^{1 / 2} \\
& =D\left(f / Q_{s_{j}}, Q_{s_{k}}\right)
\end{aligned}
$$

(where use of $M \subseteq U_{T}$ and $f \in C(\Omega)$ is made). 
With the aid of one of the triangle inequalities and of (1) we infer that

(2) $\left\|f_{s_{j}}-f_{s_{k}}\right\|_{Q} \leqslant D\left(f / Q_{s_{j}}, P_{s_{j}}\right)+D\left(f / P_{s_{j}}, P_{s_{k}}\right)+D\left(f / P_{s_{k}}, Q_{s_{k}}\right)$.

By translation invariance, since $j, k \geqslant N$, and by our choice of $P$, it follows that each term on the right hand side of (2) does not exceed $\varepsilon / 4$, whence $\left\|f_{s_{j}}-f_{s_{k}}\right\|_{Q} \leqslant \varepsilon$ is proved for $j, k \geqslant N$. Thus $Q$ is compact and we have shown that

(3) given $t_{1}, t_{2}, \ldots \in \mathbb{R}$ there is a subsequence $s_{n}=t_{j_{n}}, n=1,2, \ldots$ such that $f_{s_{1}}, f_{s_{2}}, \ldots$ is a Cauchy sequence with respect to $L_{2}\left(\Omega, \mu_{Q}\right)$ for all $Q \in M$.

It is now easy to see that (3) is even true for all $Q \in M^{*}$ with $M^{*}=\bigcup M_{t}, t \in \mathbb{R}$. Thus if $Q \in M^{*}$ then $Q=P_{t}$ for some $P \in M$ and some $t \in \mathbb{R}$, and hence $\mu_{P}=\mu_{Q}$ by $\S 2(\mathrm{~b})(3)$. Since $f_{s_{1}}, f_{s_{2}}, \ldots$ is Cauchy with respect to $L_{2}\left(\Omega, \mu_{P}\right)$, it is Cauchy with respect to $L_{2}\left(\Omega, \mu_{Q}\right)$. We thus have shown that

(4) the property in clause (3) holds for all $Q \in M^{*}$.

(S3) The proof now follows from clause (4). Thus let $t_{n} \in \mathbb{R}, n=1,2, \ldots$ be an arbitrary sequence. By (3) and (4) in (S2) there is a subsequence $s_{1}, s_{2}, \ldots$ such that $f_{s_{1}}, f_{s_{2}}, \ldots$ is a Cauchy sequence with respect to $L_{2}\left(\Omega, \mu_{P}\right)$ for all $P \in M^{*}$. Thus

$$
\lim _{N} \sup _{m, n \geqslant N} \int\left|f_{s_{n}}-f_{s_{m}}\right|^{2} d \mu_{P}=0
$$

for all $P \in M^{*}$. Since $f \in C(\Omega)$ we infer from the dominated convergence theorem that

(5) $\lim _{N} \int_{M^{*}} d \mu \sup _{m, n \geqslant N} \int\left|f_{s_{n}}-f_{s_{m}}\right|^{2} d \mu_{P}=0$

Now the supremum can be interchanged with the first integral sign without increasing the expression in (5). We thus obtain

(6) $\quad \lim \sup _{m, n \geqslant N} \int_{M^{*}} d \mu \int\left|f_{s_{n}}-f_{s_{m}}\right|^{2} d \mu_{P}=0$.

Due to the invariance of $M^{*}$ and to equation $(\beta)$ in $\S 2$ we have $\int_{\mathcal{M}^{*}} g d \mu=\int_{\mathcal{M}^{*}} d \mu \int g d \mu_{P}$ for all $g \in L_{2}(\Omega, \mu)$. Clause (6) thus reduces to

(7) $\lim \sup _{m, n \geqslant N} \int_{M^{*}}\left|f_{s_{n}}-f_{s_{m}}\right|^{2} d \mu=0$

But (7) just states that $f_{s_{n}} \chi_{M^{*}}, n=1,2, \ldots$ is a Cauchy sequence with respect to $U_{t}, t \in \mathbb{R}$. Thus $f \chi_{M^{*}}$ is compact and the theorem follows. 
We again write $\mathbf{H}(f)$ for the closed invariant subspace of $L_{2}(\Omega, \mu)$ spanned by the elements $U_{t} f, t \in \mathbb{R}$.

Corollary 1. Let $f \in C(\Omega)$ be such that there is a sequence $M_{k} \subseteq U_{T}, k=1,2, \ldots$ of closed sets which satisfy

(1) $M_{k}$ is B-stable with respect to $f$,

(2) if $P \in M_{k}$ then $P$ is compact with respect to $f$,

(3) $\lim _{k} \mu\left(M_{k}\right)=1$.

Then $U_{t}, t \in \mathbb{R}$, restricted to $\mathbf{H}(f)$, has pure point spectrum.

Proof. By (1), (2) and Theorem $1, U_{t}, t \in \mathbb{R}$ has pure point spectrum in $\mathbf{H}\left(f \chi_{M_{k}^{*}}\right), k=1,2, \ldots$ By $(3), f \chi_{M_{k}^{*}} k=1,2, \ldots$ converges to $f$ in the $L_{2}(\Omega, \mu)$-sense. Thus $U_{t}, t \in \mathbb{R}$ has pure point spectrum in $\mathbf{H}(f)$ by $[\mathbf{8}$, Chapter $10, \S 1]$.

Corollary 2. Let there be a subset $D \subseteq C(\Omega)$ which is dense in $L_{2}(\Omega, \mu)$ and such that every $f \in D$ satisfies the assumptions of Corollary 1 . Then $U_{t}, t \in \mathbb{R}$ has pure point spectrum.

Proof. By Corollary $1, U_{t}, t \in \mathbb{R}$ has pure point spectrum in $\mathbf{H}(f)$ for $f \in D$. Since $D$ is dense in $L_{2}(\Omega, \mu)$, the statement follows from [8, Chapter $\left.10, \S 1\right]$.

\section{Necessary conditions for a pure point spectrum}

In order to get necessary and sufficient conditions for a flow to have pure point spectrum we first need a converse to Corollary 1 , as follows.

THEOREM 2. Assume that for some $f \in C(\Omega), U_{t}, t \in \mathbb{R}$, restricted to $\mathbf{H}(f)$, has pure point spectrum. Then there is a sequence of closed sets $M_{k} \subseteq U_{T}, k=1,2, \ldots$ such that

(1) $M_{k}$ is B-stable with respect to $f$,

(2) if $P \in M_{k}$ then $P$ is compact with respect to $f$,

(3) $\lim _{k} \mu\left(M_{k}\right)=1$.

Proof. We proceed in steps.

(S0) Let $U_{t}, t \in \mathbb{R}$ have pure point spectrum in $\mathbf{H}(f)$ with $\lambda_{1}, \lambda_{2}, \ldots$ the list of eigenvalues and $L_{1}, L_{2}, \ldots \subseteq \mathbf{H}(f)$ the corresponding eigenspaces. By routine arguments given in [5] there is an invariant Borel set $M_{0}$ with $\mu\left(M_{0}\right)=1$ such that (a) if $P \in M_{0}$ then

$$
f_{k}(P)=\lim (2 T)^{-1} \int_{-T}^{+T} f\left(P_{t}\right) \exp \left(-i \lambda_{k} t\right) d t
$$


exists. The function $f_{k}$ is simply the projection of $f$ onto the eigenspace $L_{k}$ and for $P \in M_{0}$ we have (b) $f_{k}\left(P_{s}\right)=f_{k}(P) \exp \left(i \lambda_{k} s\right), s \in \mathbb{R}$. Since $\Omega-M_{0}$ is invariant we may put $f_{k}(P)=0$ on $\Omega-M_{0}$; equation (b) then holds for all $P \in \Omega$.

(S1) By equation $(\gamma)$ in $\S 2$ there is a set $M_{1} \subseteq M_{0} \cap U_{T}$ with $\mu\left(M_{1}\right)=1$ with the property that (c) if $P \in M_{1}$ then

$$
\lim (2 T)^{-1} \int_{-T}^{+T}\left|f-\sum_{1}^{n} f_{k}\right|^{2} d t=\int\left|f-\sum_{1}^{n} f_{k}\right|^{2} d \mu_{P}
$$

for $n=1,2, \ldots$. Since $U_{t}, t \in \mathbb{R}$ has pure point spectrum in $\mathbf{H}(f)$ it follows from (II), (III) and (IV) in $\$ 2$ that there is no loss in generality in assuming that $M_{1}$ also satisfies the property that (d) if $P \in M_{1}$ then $P$ is compact with respect to $f$.

(S2) Now take $\varepsilon>0$ arbitrary. Let $\varepsilon_{k}, k=1,2, \ldots$ be such that $\varepsilon_{k}>\varepsilon_{k+1}>0, \sum_{1}^{\infty} \varepsilon_{k}=\varepsilon$. According to Lusin's theorem [4, p. 243] there is for every $k$ a compact set $C_{k} \subseteq M_{1}$ with the following properties: (e) $f_{k}$ is continuous and hence uniformly continuous on $C_{k}$ and (f) $\mu\left(C_{k}\right)>1-\varepsilon_{k}$. Thus $C=\bigcap C_{k}$ is a compact set such that $\left(\mathrm{g}_{1}\right) f_{k}, k=1,2, \ldots$ is uniformly continuous on $C$, $\left(\mathrm{g}_{2}\right) C \subseteq M_{1}$ and $\left(\mathrm{g}_{3}\right) \mu(C) \geqslant 1-\varepsilon$.

(S3) Since $f_{k}$ is the projection of $f$ onto the eigenspace $L_{k}$ of $\lambda_{k}$ we infer that (h) $\lim _{n} \int\left|f-\sum_{1}^{n} f_{k}\right|^{2} d \mu=0$. By combining (h) with equation $(\beta)$ of $\S 2$ we obtain that (i) $\lim _{n} \int d \mu \int\left|f-\sum_{1}^{n} f_{k}\right|^{2} d \mu_{P}=0$. Hence there is a sequence $n_{j}, j=1,2, \ldots$ and a set $M_{2} \subseteq M_{1}$ with $\mu\left(M_{2}\right)=1$ such that $\lim _{j} \int\left|f-\sum_{1}^{n_{j}} f_{k}\right|^{2} d \mu_{P}=0$ for $P \in M_{2}$. By Egoroff's theorem [4, p. 88] there is a Borel set $M_{2}^{\prime} \subseteq M_{2}$ with $\mu\left(M_{2}^{\prime}\right)>1-\varepsilon / 2$ in which, for every $\delta>0$, there is a $j_{0}$ such that $j \geqslant j_{0}$ implies that $\int\left|f-\sum_{1}^{n_{j}} f_{k}\right|^{2} d \mu_{P} \leqslant \delta$ for all $P \in M_{2}^{\prime}$. Since $\mu$ is regular there is a closed set $C^{\prime} \subseteq M_{2}$ with $\mu\left(C^{\prime}\right) \geqslant 1-\varepsilon$ having the property that $(\mathrm{j})$ on $C^{\prime}$ the sequence $\int\left|f-\sum_{1}^{n_{j}} f_{k}\right|^{2} d \mu_{P}, j=1,2, \ldots$
converges uniformly to zero.

(S4) Now consider the set $A=C \cap C^{\prime}$ with $C$ as in (S2) and $C^{\prime}$ as in (S3). The set $A$ is a compact subset of $U_{T}$ with measure $\mu(A) \geqslant 1-2 \varepsilon$ and the property that if $P \in A$ then $P$ is compact with respect to $f$. The theorem is then essentially proved if we can show that $A$ is $B$-śtable with respect to $f$. In order to accomplish this last step we first claim that $A$ is $B$-stable with respect to each $f_{k}$. Now $f_{k}$ is uniformly continuous on $A$ by $\left(\mathrm{g}_{1}\right)$ and $\left(\mathrm{g}_{2}\right)$, and because $A \subseteq C$. Thus for $\varepsilon^{\prime}>0$ there is a $\delta>0$ such that if points $P, Q \in A$ satisfy $d(P, Q) \leqslant \delta$ then $\left|f_{k}(P)-f_{k}(Q)\right|<\varepsilon^{\prime}$. Since equation (b) in (S0) holds on the set $A$ we find the relations

$$
D\left(f_{k} / P, Q\right)^{2}=\lim (2 T)^{-1} \int_{-T}^{+T}\left|f_{k}\left(P_{t}\right)-f_{k}\left(Q_{t}\right)\right|^{2} d t=\left|f_{k}(P)-f_{k}(Q)\right|^{2} \leqslant\left(\varepsilon^{\prime}\right)^{2} .
$$


Thus $D\left(f_{k} / P, Q\right) \leqslant \varepsilon^{\prime}$ provided that $d(P, Q) \leqslant \delta$ and $P, Q \in A$. Therefore $A$ is indeed $B$-stable with respect to $f_{k}$. From the second triangle inequality in the remark after Definition 1 it follows that if a set $M$ is $B$-stable with respect to $g$ and $h$ then it is $B$-stable with respect to $g+h$. Thus we find that the set $A$ is $B$-stable with respect to $G_{j}=\sum_{1}^{n_{j}} f_{k}, j=1,2, \ldots$ It remains to prove the $B$-stability of $A$ with respect to $f$. To this end we take a $j$ so large that $\left\{\int\left|f-\sum_{1}^{n_{j}} f_{k}\right|^{2} d \mu_{P}\right\}^{1 / 2} \leqslant \varepsilon^{\prime} / 3$ for all $P \in A((\mathrm{~S} 3)(\mathrm{j}))$. For $\varepsilon^{\prime}$ and $G_{j}=\sum_{1}^{n_{j}} f_{k}$ we determine a $\delta>0$ such that $P, Q \in A$ and $d(P, Q) \leqslant \delta$ imply that $D\left(G_{j} / P, Q\right) \leqslant \varepsilon^{\prime} / 3$. Now we observe that the following triangle inequality holds:

$\begin{aligned}(k) D(f / P, Q) \leqslant \lim \left\{(2 T)^{-1} \int_{-T}^{+T}\left|f\left(P_{t}\right)-G_{j}\left(P_{t}\right)\right|^{2} d t\right\}^{1 / 2}+D\left(G_{j} / P, Q\right) & \\ + & \lim \left\{(2 T)^{-1} \int_{-T}^{+T}\left|G_{j}\left(Q_{t}\right)-f\left(Q_{t}\right)\right|^{2} d t\right\}^{1 / 2} .\end{aligned}$

By (S1)(c) the first and third terms are equal to

$$
\left\{\int\left|f-G_{j}\right|^{2} d \mu_{P}\right\}^{1 / 2}
$$

and

$$
\left\{\int\left|f-G_{j}\right|^{2} d \mu_{Q}\right\}^{1 / 2}
$$

respectively and hence are not larger than $\varepsilon^{\prime} / 3$ according to our choice of $j$; the second term does not exceed $\varepsilon^{\prime} / 3$ since $d(P, Q) \leqslant \delta$. Therefore we have $D(f / P, Q) \leqslant \varepsilon^{\prime}$. To sum up, given $\varepsilon$ we have found a closed set $A \subseteq U_{T}$ with $\mu(A) \geqslant 1-2 \varepsilon$ and the properties (1) if $P \in A$ then $P$ is compact with respect to $f$ and (2) $A$ is $B$-stable with respect to $f$. From this the theorem follows immediately.

Corollary 1. Assume that $f \in C(\Omega)$. Then $U_{t}, t \in \mathbb{R}$ has pure point spectrum in $\mathbf{H}(f)$ if and only if there is a sequence of closed sets $M_{k}, k=1,2, \ldots$ which satisfy

(a) if $P \in M_{k}$ then $f\left(P_{t}\right), t \in \mathbb{R}$ is Besicovitch almost periodic,

(b) $M_{k}$ is B-stable with respect to $f$ and

(c) $\lim _{k} \mu\left(M_{k}\right)=1$.

Proof. Let a sequence $M_{k}, k=1,2, \ldots$ of closed sets satisfy (a), (b) and (c). Since the measure $\mu$ is regular and $\mu\left(U_{T}\right)=1$ it follows from (c) and from $\$ 2$ (III) that there is a sequence of closed sets $M_{k}^{\prime}, k=1,2, \ldots$ which satisfy (1) $M_{k}^{\prime} \subseteq M_{k} \cap U_{T}$, (2) $\mu\left(M_{k}-M_{k}^{\prime}\right) \leqslant 2^{-k}$, and (3) if $P \in M_{k}^{\prime}$ then $P$ is compact with respect to $f$. Thus $M_{k}^{\prime}, k=1,2, \ldots$ clearly satisfies the conditions of Theorem 2 with respect to $f$. Therefore $U_{t}, t \in \mathbb{R}$ has pure point spectrum in $\mathbf{H}(f)$. If, conversely, 
$U_{t}, t \in \mathbb{R}$ has pure point spectrum in $\mathbf{H}(f)$ then there is a sequence $M_{k}, k=1,2, \ldots$ of closed sets which satisfy (1), (2) and (3) of Theorem 2. By arguments as above we then find closed sets $M_{k}^{\prime}, k=1,2, \ldots$ which satisfy $\left(1^{\prime}\right) \quad M_{k}^{\prime} \subseteq M_{k}$, (2') $\mu\left(M_{k}-M_{k}^{\prime}\right) \leqslant 2^{-k}$ and $\left(3^{\prime}\right)$ if $P \in M_{k}^{\prime}$ then $f\left(P_{t}\right), t \in \mathbb{R}$ is Besicovitch almost periodic. The sequence $M_{k}^{\prime}, k=1,2, \ldots$ then clearly satisfies (a), (b) and (c) of the corollary.

Corollary 2. $U_{t}, t \in \mathbb{R}$ has pure point spectrum in $L_{2}(\Omega, \mu)$ if and only if there is a denumerable subset $D \subseteq C(\Omega)$, dense in $C(\Omega)$ with respect to the supremum norm sup $|f(P)|$, such that for every $f \in D$ there are closed sets $M_{k}, k=1,2, \ldots$ which satisfy $(a),(b)$ and $(c)$ of Corollary 1.

Proof. The group $U_{t}, t \in \mathbb{R}$ has pure point spectrum in $L_{2}(\Omega, \mu)$ if and only if for every $f \in L_{2}(\Omega, \mu)$ we have that (1) $U_{t}, t \in \mathbb{R}$ has pure point spectrum in $\mathbf{H}(f)$. On the other hand, the set of elements $f$ in $L_{2}(\Omega, \mu)$ having property (1) form a closed linear subspace of $L_{2}(\Omega, \mu)[8$, Chapter $10, \S 1]$. Since $C(\Omega)$ is dense in $L_{2}(\Omega, \mu)$, the statement follows from Corollary 1.

\section{Flows in $\mathbb{R}^{n}$}

The necessary and sufficient conditions mentioned in Corollaries 1 and 2 to Theorem 2 do not have the form described in the introduction. In order to obtain statements of this form we have to restrict the permitted topological spaces. Henceforth we assume that

(A) $\Omega$ is a closed subset of $\mathbb{R}^{n}$.

On $\Omega$ we have a distinguished set of continuous functions $g_{j} \in C(\Omega), j=1,2, \ldots, n$ which associate to every point $P=\left(x_{1}, \ldots, x_{n}\right)$ of $\Omega$ its $j$-th coordinate $g_{j}(P)=x_{j}$. We assume that the metric $d$ on $\Omega$ is the Euclidean metric given by $d(P, Q)=\left\{\sum_{1}^{n}\left(g_{j}(P)-g_{j}(Q)\right)^{2}\right\}^{1 / 2}$. We introduce the following distance between the points $P, Q \in \Omega$ :

$$
\rho(P, Q)=\lim \sup _{T}\left\{(2 T)^{-1} \int_{-T}^{+T} d\left(P_{t}, Q_{t}\right)^{2} d t\right\}^{1 / 2} .
$$

Finally we need a variant notion of $B$-stability.

Definition 3. A set $M$ is called $B^{*}$-stable if for every $\varepsilon>0$ there is a $\delta>0$ such that $P, Q \in M$ and $d(P, Q) \leqslant \delta$ imply that $\rho(P, Q) \leqslant \varepsilon$.

In preparation for Theorem 3 we need a lemma on Besicovitch almost periodic functions whose proof is completely routine and therefore omitted.

Lemma 1. Let $f(t), g(t), t \in \mathbb{R}$ be bounded Besicovitch almost periodic functions $(|f(t)|,|g(t)| \leqslant K)$. Then $f+g$ and $f g$ are Besicovitch almost periodic. 
Lemma 2. Let $U_{t}, t \in \mathbb{R}$ have pure point spectrum in $L_{2}(\Omega, \mu)$. Then there is a sequence of closed sets $M_{k}, k=1,2, \ldots$ with the properties that

(1) if $P \in M_{k}$ then $g_{j}\left(P_{t}\right), t \in \mathbb{P}$ is Besicovitch almost periodic for $j=1,2, \ldots, n$,

(2) $M_{k}$ is $B^{*}$-stable, and

(3) $\lim _{k} \mu\left(M_{k}\right)=1$.

Proof. Since $U_{t}, t \in \mathbb{R}$ has pure point spectrum in $L_{2}(\Omega, \mu)$ it has pure point spectrum in each subspace $\mathbf{H}\left(g_{j}\right) \subseteq L_{2}(\Omega, \mu), j=1,2, \ldots, n$. By Corollary 1 to Theorem 2 there is a sequence of closed sets $M_{k}, k=1,2, \ldots$ such that (a) if $P \in M_{k}$ then $g_{j}\left(P_{t}\right), t \in \mathbb{B}$ is Besicovitch almost periodic for $j=1,2, \ldots, n$, (b) $M_{k}$ is $B$-stable with respect to $g_{j}, j=1,2, \ldots, n$, (c) $\lim _{k} \mu\left(M_{k}\right)=1$.

We now prove part (2). Fix $M_{k}$ and $\varepsilon>0$. By (b) there is a $\delta>0$ such that $d(P, Q) \leqslant \delta$ implies that $D\left(g_{j} / P, Q\right) \leqslant \varepsilon / n, j=1,2, \ldots, n$. A straightforward computation then yields

$$
\rho(P, Q)=\lim \sup _{T}\left\{(2 T)^{-1} \int_{-T}^{+T} \sum_{1}^{n}\left(g_{j}\left(P_{t}\right)-g_{j}\left(Q_{t}\right)\right)^{2} d t\right\}^{1 / 2} \leqslant \sum_{1}^{n} D\left(g_{j} / P, Q\right) \leqslant \varepsilon,
$$

and the proof is complete.

We need also the converse of Lemma 2.

Lemma 3. Let there be a sequence of closed sets $M_{k}, k=1,2, \ldots$ such that

(1) if $P \in M_{k}$ then $g_{j}\left(P_{t}\right), t \in \mathbb{R}$ is Besicovitch almost periodic for $j=1, \ldots, n$,

(2) $M_{k}$ is $B^{*}$-stable, and

(3) $\lim _{k} \mu\left(M_{k}\right)=1$.

Then $U_{t}, t \in \mathbb{R}$ has pure point spectrum in $L_{2}(\Omega, \mu)$.

Proof. We make use of Corollary 2 to Theorem 2. For the denumerable, dense set $D \subseteq C(\Omega)$ we define $D$ thus: $f \in D$ if and only if there is a polynomial $\phi\left(x_{1}, \ldots, x_{n}\right)$ in the variables $x_{1}, \ldots, x_{n}$ with rational coefficients such that $f(P)=\phi\left(g_{1}(P), \ldots, g_{n}(P)\right), P \in \Omega$. The set $D$ is then clearly denumerable and dense in $C(\Omega)$ with respect to the supremum norm. Since for each $P \in M_{k}$ the functions $g_{j}\left(P_{t}\right), t \in \mathbb{R}$ are Besicovitch almost periodic for $j=1, \ldots, n$ it follows from Lemma 1 that if $P \in M_{k}$ and $f \in D$ then $f\left(P_{t}\right), t \in \mathbb{R}$ is Besicovitch almost periodic. Since $\lim \mu\left(M_{k}\right)=1$ by assumption, all that remains is to verify (b) in Corollary 1 to Theorem 1 for $f \in D$. To this end we note that each function $f \in D$ is Lipschitz continuous, that is, there is a $C_{f}>0$ such that $|f(P)-f(Q)| \leqslant C_{f} d(P, Q)$. Now we fix an $M_{k}$ and an $\varepsilon>0$. By assumption (2) there is a $\delta>0$ such that $P, Q \in M_{k}$ and 
$d(P, Q) \leqslant \delta$ imply that $\rho(P, Q) \leqslant C_{f}^{-1} \varepsilon$. For $P, Q \in M_{k}$ and $d(P, Q) \leqslant \delta$ we then have

$$
D(f / P, Q) \leqslant \lim \sup _{T}\left\{(2 T)^{-1} \int_{-T}^{+T} C_{f}^{2} d\left(P_{t}, Q_{t}\right)^{2} d t\right\}^{1 / 2}=C_{f} \rho(P, Q) \leqslant \varepsilon .
$$

Hence $M_{k}$ is $B$-stable with respect to $f$; this proves the lemma.

THEOREM 3. Under the assumptions of this section, $U_{t}, t \in \mathbb{R}$ has pure point spectrum in $L_{2}(\Omega, \mu)$ if and only if there are closed sets $M_{k}, k=1,2, \ldots$ such that

(1) if $P \in M_{k}$ then $g_{j}\left(P_{t}\right), t \in \mathbb{R}$ is Besicovitch almost periodic for $j=1, \ldots, n$,

(2) $M_{k}$ is $B^{*}$-stable, and

(3) $\lim _{k} \mu\left(M_{k}\right)=1$.

\section{Remarks}

(A) The decomposition theory of Bogoljubov-Krylov is used here in a more general form than as it is in the literature. In $[2,11]$ it is assumed that the flow $S_{t}, t \in \mathbb{R}$ satisfies

(i) $S_{t}(P)$ is simultaneously continuous in $P, t$.

In this paper we only assume that

(ii) the mapping $\mathbb{R} \times \Omega \rightarrow \Omega$ which associates $S_{t}(P)$ with $P, t$ is measurable.

An inspection of [11] shows that clauses (1)-(4) in $\$ 2$ depend on (i) in only one case, namely the proof that the measure $\mu_{P}$ defined by

$$
\int f d \mu_{P}=\lim (2 T)^{-1} \int_{-T}^{+T} f\left(P_{t}\right) d t, f \in C(\Omega),
$$

is indeed invariant. A proof of this fact requires an elementary but somewhat subtle approximation argument and a shrinking of $U_{T}$ (some transitive points in the sense of [11] have to be omitted). The full details are worked out in [7] where the theory of Bogoljubov-Krylov is generalized to arbitrary, measure preserving abelian groups. An alternative approach would have been to work with the decomposition theory of Rohlin [13]. However this would have forced the introduction of a number of notions centering around the concept of measurable partition, making the paper considerably more involved.

(B) As to the problem, treated here, of characterizing the pure point spectrum of a flow in "dynamical" terms, there is a remark in [16] to the effect that the flow $\left(\Omega, S_{t}, t \in \mathbb{R}, \mu\right)$ has pure point spectrum if and only if for every $f \in L_{2}(\Omega, \mu)$ the function $f\left(P_{t}\right), t \in \mathbb{R}$ is Besicovitch almost periodic for almost all $P$. However this 
statement is only true if the flow is ergodic as simple counterexamples show (consider, for example, the flow $T_{2}$ in the introduction). In the general case one has to replace the ergodicity requirement by another global condition such as $B$-stability. In this connection another well-known result has to be mentioned. Call a flow $F=\left(\Omega, S_{t}, t \in \mathbb{R}, \mu\right)$ uniformly Ljapounov stable if it satisfies the condition that

(iii) for $\varepsilon>0$ there is a $\delta>0$ such that for all $P, Q$ with $d(P, Q) \leqslant \delta$ we have $d\left(P_{t}, Q_{t}\right) \leqslant \varepsilon$ for all $t \in \mathbb{R}$.

It is shown at different places that (iii) is equivalent to the existence of denumerably many eigenvalues $\lambda_{1}, \lambda_{2}, \ldots$ with the property that

(iv) for $f \in C(\Omega)$ there exist eigenfunctions $\phi_{n} \in C(\Omega), n=1,2, \ldots$ (with $\left.\phi_{n}\left(P_{t}\right)=\phi_{n}(P) \exp \left(i \lambda_{n} t\right)\right)$ and coefficients $a_{j}^{n}, j=1, \ldots, n$ such that $\sup _{P}\left|f(P)-\sum_{1}^{n} a_{j}^{n} \phi_{j}(P)\right|$ tends to zero as $n \rightarrow \infty$.

For details we refer to [6]. The results of this paper (notably Theorem 3) may be considered as a generalisation of this situation to a much wider class of flows.

(C) Trivial examples which illustrate Theorem 3 are easily obtained by proceeding as in the introduction, namely by considering various kinds of rotations and mixtures of rotations. The question arises whether there are more sophisticated examples to which Theorem 3 is applicable. This is indeed the case. In [9] N. Kolmogorov constructed a particular flew $F_{0}=\left(\Omega, S_{t}, t \in \mathbb{R}, \mu\right)$ which has the properties that (1) $\Omega$ is the 2-torus, (2) the flow $S_{t}, t \in \mathbb{R}$ is generated by analytical differential equations, and (3) the induced unitary group $U_{t}, t \in \mathbb{R}$ has pure point spectrum, is ergodic and has no other continuous eigenfunctions besides the constants. Thus the flow $F_{0}$ is certainly not uniformly Ljapounov stable by the above remarks. However Theorem 3 is applicable, and so it follows that $F_{0}$ still has some Ljapounov like stability properties, namely those described by Definition 3 and Theorem 3, clauses (2) and (3). The details of the Kolmogorov construction are described in [15, lecture 11]; variations of the construction and extensions to higher dimensions are given in [10]. Theorem 3 can of course be used in the negative direction, namely to infer for flows which are known not to have pure point spectrum that the trajectories do not exhibit the stability properties required by Theorem 3 .

(D) As the referee pointed out, there is a stability notion which is equivalent to $B^{*}$-stability and which has been considered at several places in the literature; this is the concept of mean $L$-stability (S. Fomin [3], J. C. Oxtoby [12], J. Auslander [1]). In order to adapt this concept to the framework of $\$ 5$ we extend the definition in [12, p. 127], given for discrete time systems, to the continuous case. With every Borel set $E \subseteq \mathbb{B}$ we associate its upper density $\delta^{*}(E)=\lim \sup _{T}(2 T)^{-1} \int_{-T}^{+T} \chi_{E}(t) d t$, with $\chi_{E}$ the characteristic function of $E$. A Borel set $M \subseteq \Omega$ is called mean $L$-stable if for every $\varepsilon$ there is $\delta$ such that $P, Q \in M$ and $d(P, Q) \leqslant \delta$ imply that $d\left(P_{t}, Q_{t}\right) \leqslant \varepsilon$ for all $t \in \mathbb{R}$ with the possible exception of a set $E \subseteq \mathbb{R}$ (depending on $P, Q$ ) of mean upper density at most $\varepsilon$. As the referee observed, mean $L$-stability and $B^{*}$-stability are 
equivalent. We prove one direction of this equivalence. An easy calculation shows that

$$
\lim \sup _{T}(2 T)^{-1} \int_{-T}^{+T} d\left(P_{t}, Q_{t}\right) d t \leqslant \rho(P, Q)
$$

where $d(P, Q)$ and $\rho(P, Q)$ are defined as in $\S 5$. Now let $M$ be $B^{*}$-stable. Thus to $\varepsilon>0$ there is a $\delta>0$ with $\rho(P, Q) \leqslant \varepsilon^{2}$ whenever $P, Q \in M$ and $d(P, Q) \leqslant \delta$. By the inequality displayed above we find that

$$
\lim \sup _{T}(2 T)^{-1} \int_{-T}^{+T} d\left(P_{t}, Q_{t}\right) d t \leqslant \varepsilon^{2}
$$

It then follows that the set $E=\left\{t: d\left(P_{t}, Q_{t}\right) \geqslant \varepsilon\right\}$ has mean upper density at most $\varepsilon$. This proves that $B^{*}$-stability implies mean $L$-stability. The proof of the converse is similar.

(E) There are some open questions which suggest themselves. For instance it would be important to know if for ergodic flows Theorem 3 still holds if only conditions (2) and (3) are retained. A related question (suggested by the referee) asks whether a $B^{*}$-stable (or, equivalently, a mean $L$-stable) minimal set has pure point spectrum. Briefly, the main question is whether under additional conditions one can eliminate every reference to Besicovitch almost periodic functions. We know of no proofs for, nor counterexamples to, this conjecture.

\section{References}

1. J. Auslander, 'Mean L-stable systems', Illinois J. Math., 3 (1959), 566-579.

2. N. Bogoljubov and N. Krylov, 'Théorie de la mesure dans la mécanique', Ann. of Math., 38 (1937), 65-113.

3. S. Fomin, 'On dynamical systems with pure point spectrum', Dokl. Akad. Nauk. SSSR (NS), 77 (1951), 29-32.

4. P. R. Halmos, Measure theory (Van Nostrand, New York, 1960).

5. E. HoPf, Ergodentheorie (Springer, Berlin, 1937).

6. K. JACOBS, Neuere Methoden und Ergebnisse der Ergodentheorie (Springer, Berlin, 1960).

7. U. KAESER, Ergodentheorie und individuelle Masse für Abelsche Gruppen (Diplomarbeit, Basel, 1979).

8. T. KATO, Perturbation theory for linear operators (Springer, Berlin, 1966).

9. A. N. Kolmogorov, 'On dynamical systems with an intergral invariant on the torus', Dokl. Akad. Nauk. SSSR (NS), 93 (1953), 763-766.

10. D. A. LIND, 'Spectral invariants and smooth ergodic theory', Lecture Notes in Physics 38 (1974), pp. 296-309.

11. V. NemitKII and V. StePanov, Qualitative theory of differential equations (Princeton University Press, Princeton, 1974).

12. J. C. Oxтовy, 'Ergodic sets', Bull. Amer. Math. Soc., 58 (1952), 116-136.

13. V. Rohlin, 'Lectures on entropy theory of transformations with an invariant measure', Russian Math. Surveys, 22 (1967), 1-52.

14. B. SCARPELlinl, 'Fourier analysis on dynamical systems', J. Differential Equations, 28 (1978), 309-326. 
15. Y. G. SinAl, Introduction to ergodic theory, Mathematical Notes (Princeton University Press, Princeton, 1977).

16. N. Wiener and N. WinTNER, 'On the ergodic dynamics of almost periodic systems', Amer. J. Math., 63 (1941), 794-824.

\section{Mathematics Institute, University of Basel, 4051 Basel, \\ Rheinsprung 21, Switzerland.}

